\title{
Breast cancer screening of women aged 70-74 years: results from a natural experiment across Australia.
}

Carolyn Nickson ${ }^{1}$, Kate E Mason ${ }^{1}$, Anne M Kavanagh ${ }^{1}$

1. Centre for Women's Health, Gender and Society, Melbourne School of Population and Global Health, University of Melbourne

Corresponding author: Carolyn Nickson, email cnickson@unimelb.edu.au, phone +61 383440765 , fax +61 393479824

Keywords: Breast cancer, screening, age. 


\section{Abstract}

\section{Objective}

To what extent does screening women aged 70-74 result in smaller, earlier stage breast cancers in the population?

\section{Design}

An ecological study comparing state and territory level screening participation rates and cancer outcomes, using Poisson models weighted by population size and allowing for clustering by state/territory and calendar effects.

\section{Setting}

BreastScreen Australia and cancer registry data, 1996-2005.

\section{Participants}

Australian resident women aged 70-74 years.

\section{Main outcome measures}

State and territory invasive breast cancer incidence (all cancers, large (>15mm) cancers, nodal involvement (yes/no)), according to screening participation rates.

\section{Results}

With each $10 \%$ absolute increase in screening participation, there was no significant difference in cancer incidence, but the incidence of large cancers was $8 \%$ lower (IRR $=0.92,95 \%$ CI 0.90 to $0.94, \mathrm{p}<0.001$ ). We find some evidence that fewer women would be expected to have positive axillary lymph nodes.(IRR $0.97,95 \%$ CI 0.95 to $0.99, \mathrm{p}=0.004$ ), but this estimate was sensitive to assumptions regarding missing data. 


\section{Conclusions}

Increased mammographic screening of women aged 70-74 years reduces the incidence of large (>15mm) cancers - and possibly cancers with nodal involvement - without a concomitant increase in overall cancer incidence. 


\section{Introduction}

There is a lack of evidence regarding the optimal age at which to cease mammographic screening for breast cancer. The International Association for Research in Cancer describes the current recommended upper age limit of 69 years as arbitrary [1], and there is a lack of robust evidence about whether to screen women aged 70-74 years because trials did not include this age group in sufficient numbers [2]. This occurred for a number of reasons, including assumptions about competing mortality risks, the costs attached to screening and treatment versus years of life lost, reduction in quality of life associated with breast cancer diagnosis and treatment, and an expectation of lower participation by older women [1]. On the basis of the trial evidence, most screening programs have targeted women aged less than the 70 years [3]. Thus, despite significant cancer incidence in this age group (in Australia, 30 cancer diagnoses per 10,000 women aged 70-74 compared to 35 in women aged 65-69 [4]), it has not been possible to obtain evidence from observational studies to assess the potential benefits of extending the age range for screening.

The BreastScreen Australia program has since 1991 offered free, population-based biennial mammographic breast cancer screening to women from the age of 40 years [5]. Women aged 50-69 years are targeted for screening through mailed invitation upon reaching 50 years of age (via electoral roll registrations, which include over $95 \%$ of the eligible population [6]), mailed re-invitation to repeat screens, and media campaigns directed at this age group [7]. The target age range was recently extended to 74 years [8]. Between 1996 and 2005 there was substantial variation in participation by women aged 70-74 years because some state and territory BreastScreen services elected to invite them to subsequent-round screening by mail. For example, in 2004-2005, despite similar participation rates by women aged 65-69 years in all states and 
territories, participation by women aged $70-74$ years averaged $52 \%$ in jurisdictions where they were re-invited to subsequent screening rounds by mail, and 24\% where they were not [9] (Table 1).

This variation in screening participation has provided a 'natural experiment' from which the effectiveness of screening women aged 70-74 years can be evaluated. Since screening aims to detect breast cancers earlier, cancers diagnosed in states and territories with higher rates of screening participation should tend to be smaller, with less nodal involvement; this is important in Australia where women in their seventies have poorer survival if their cancers are large or node-positive [10]. Breast cancer incidence would not necessarily be higher in women aged 70-74 with increased screening, because nearly all (approximately 99\%) of BreastScreen participants aged 70-79 years have been screened some time previously [11] and 70\% have been screened within the previous 27 months [12], avoiding any significant short-term peak in incidence expected with prevalent-round screening.

This ecological study used Australian screening and cancer registry data from 1996-2005 to assess whether screening participation was associated with state-level incidence of invasive cancers; a decline in large $(>15 \mathrm{~mm})$ breast cancers; and a reduction in cancers with nodal involvement.

\section{Methods}

\section{Data collection}


BreastScreen participation data (the number of women screened and female population figures) was collected according to state/territory, fiveyear age group and two-year calendar period for the years 1996-2005 from annual BreastScreen Australia Monitoring Reports [9,13-17].

Data on cancer incidence, size and nodal involvement was requested from all state and territory cancer registries, by five-year age group and year, for the period 1996-2005. We excluded the Northern Territory because there were not a sufficient number of cancers due to the small population. Cancer incidence data (8,743 cancers in total) were obtained from all approached state and territory cancer registries except South Australia. Only some registries routinely collected data on size and nodal status, [7]; information on both was provided by New South Wales (1997), Queensland (1996-2004), Tasmania (1996-2004), Victoria (1996-2004) and Western Australia (1996-2005). The Australian Capital Territory did not collect this information for any year. Because there was missing data on size and nodal status, we conducted a range of sensitivity analyses to evaluate the potential bias that might arise our because of these missing data.

\section{Analysis}

For all analyses, calendar time was aggregated into two-year periods because screening participation was reported only by two-year period.

\section{Screening participation and breast cancer incidence}

To assess the association between screening participation and invasive breast cancer incidence, a Poisson model was fitted for states and territories with available data on size and nodal status, where the predictor term was screening participation, the outcome was the number of 
cancers diagnosed, and the model weight was population size. Screening participation was fitted as a proportion divided by ten; this meant that the effect size indicated the proportional change in cancer incidence with each $10 \%$ absolute increase in screening participation.

The female population aged 70-74 years was fitted as the model weight. The model accounted for clustering by state/territory. Where only single years of cancer data were available, outcomes were compared to screening participation for the two-year period including that year. We tested whether calendar time (two-year calendar period fitted as a linear term) was statistically significant $(\mathrm{P}<0.05)$; because it was not significant in any of the models it was not included in the final models.

The same regression model structure was used to examine the incidence of large cancers and cancers with nodal involvement. To account for missing information on tumour size and nodal involvement within units of analysis (state/territory and two-year period), each analysis unit's population size was weighted according to the proportion of cancers for which the tumour characteristic was known. For example, if for some two-year period and state/territory tumour size was recorded for $70 \%$ of all invasive cancers at the cancer registry, fitted population for that twoyear period and state/territory was scaled to $70 \%$ of the observed population size. This ensured that the observed rates of small cancers were fitted while statistically powering the Poisson model only to the actual number of cancers for which the outcome of interest was known.

\section{Sensitivity analyses}

For states/territories and calendar periods with some available size or nodal status information, several assumptions about missing information were tested to assess the possible effects of bias due to missing data. For tumour size we tested two extreme assumptions: 
1. All cancers missing tumour size information were large $(>15 \mathrm{~mm})$,

2. All cancers missing tumour size information were small $(\leq 15 \mathrm{~mm})$.

Similarly, for nodal status we tested the assumptions:

1. All cancers missing information on nodal status had nodal involvement.

2. All cancers missing information on nodal status no nodal involvement.

Models of these assumptions were weighted to ensure suitable statistical power (refer to Supplemental Materials for detailed methods).

The primary analysis was restricted to states/territories and years for which tumour size and nodal involvement was known (the 'restricted dataset').

It is possible that the associations between screening participation rates and the incidence of large and node positive cancers were different for the states and territories that collected this information. To assess whether this was likely, we compared the association between participation and cancer incidence using the restricted dataset to the association found for all years and states/territories for which breast cancer incidence was known (the 'full dataset'). We reasoned that if these associations were similar, findings on the association between participation and tumour size/nodal involvement could be generalised to the total population of Australian women aged 70-74 during this time period.

Analyses were conducted using Stata 11.0 (StataCorp., College Station, TX). This study was approved by the BreastScreen Victoria Research and Evaluation Committee in 2007. 


\section{Results}

\section{Screening participation and breast cancer incidence}

The restricted dataset included a total of 5,437 cancers. Of these, $2,323(43 \%)$ were recorded as large, $2,424(45 \%)$ as small and $720(13 \%)$ were missing information on tumour size. In terms of nodal involvement, 1,351 (25\%) were recorded as node-positive, 2,730 (50\%) as node-negative, and 1,356 (25\%) were missing information on nodal involvement. Two-yearly cancer incidence by state/territory ranged from 22 to 38 per 10,000 women, and screening participation ranged from $15 \%$ to 53\%. Detailed figures for the most recent period are shown in Table 1 . More detailed data can be obtained from the authors on request.

There was no evidence that invasive breast cancer incidence was associated with participation (IRR=1.01, 95\%CI 0.99 to 1.03 (Table 2 )) - a finding consistent with minimal overdiagnosis (screen-detection of cancers that would never present clinically). This result was the same using the full dataset $(8,743$ cancers) to assess generalisability (IRR=1.01, 95\% CI (1.00 to 1.02), $\mathrm{p}=0.156$ ).

Population-level incidence of large cancers among women aged $70-74$ years was on average $8 \%$ lower with each $10 \%$ absolute increase in screening participation $(\mathrm{IRR}=0.92,95 \% \mathrm{CI}(0.90$ to 0.94$), \mathrm{p}<0.001)$. Since we use a log-linear regression model this means, for example, if participation increased from $50 \%$ to $60 \%$, the population incidence of large breast cancers would be expected to reduce by a proportion of $8 \%$ (IRR=0.92), and if participation increased from $50 \%$ to $70 \%$, the population incidence of large breast cancers would be expected to reduce by a 
proportion of $15 \%\left(\right.$ IRR $\left.=0.92^{2}=0.85\right)$. The association was similar if all cancers missing information on size were assumed to be small or, alternatively, assumed to be large (Table 2).

Screening participation was associated with a $3 \%$ proportional reduction in the incidence of cancers with nodal involvement with each $10 \%$ absolute increase in screening participation ( $I R R=0.97$ ( 0.95 to 0.99$) \mathrm{p}=0.004$ ). This means, for example, if participation increased from $50 \%$ to $60 \%, 3 \%$ fewer women would be expected to have positive axillary lymph nodes (IRR=0.97), and if participation increased from $50 \%$ to $70 \%$, $6 \%$ fewer women would be expected to have positive axillary lymph nodes (IRR $=0.97^{2}=0.94$ ). Sensitivity analyses indicated that this effect size would increase slightly (to IRR $=0.95$ with each $10 \%$ increase in participation) if all cancers missing information on nodal status were assumed to have nodal involvement; however, there was no evidence of a reduction if we assumed that all cancers missing information on nodal status had no nodal involvement (Table 2).

\section{Discussion}

This study shows that in the Australian population-based mammographic screening program, higher screening participation by women aged 7074 years was associated with a higher incidence of cancers with a better prognosis (smaller and node-negative cancers) without a concomitant rise in overall breast cancer incidence. With a $10 \%$ absolute increase in screening participation it is estimated that $8 \%$ fewer women would be diagnosed with large cancers. There is some evidence that fewer women (around 3\%) would be expected to have positive axillary lymph nodes, but this estimate was sensitive to assumptions regarding missing data. 
A strength of this study is that it utilised a 'natural experiment' in a large population with a well-organised population breast cancer screening program and good-quality information on screening participation and cancer incidence.

As a limitation, this ecological study relied on population-level data, with no individual-level information on screening participation or cancer outcomes. Therefore we were unable to estimate cancer outcomes for screened versus unscreened women. An observational study such as a cohort study would generate this important evidence.

The finding of fewer large cancers with increased participation was robust to different assumptions about missing data. However, it is likely that in our primary analysis we overestimated the association between screening participation and node-positive cancers; our sensitivity analysis where we assumed that all cancers missing nodal information were non-nodal is most likely closer to the truth, because nodal information would be missing when surgeons did not sample the nodes on the basis that nodal involvement was unlikely. As expected, we observed no increase in population cancer incidence with increased participation; screening in this age group appears to advance cancer detection predominantly through earlier detection of incident cancers.

The known poorer survival for Australian women aged 70-74 years with larger cancers at diagnosis suggests that the effects of screening observed in this study would lead to a reduction in mortality. This is consistent with evidence from a recent case-control study conducted in South Australia that found a 57\% reduced risk of breast cancer mortality for BreastScreen participants aged 70-79 years at diagnosis $(\mathrm{OR}=0.43$ $(0.25,0.73))[18]$. While the Swedish Two County Trial showed no significant mortality reduction for women aged 70-74 years on entry, [19] it is difficult to compare these results to a population program like BreastScreen Australia because women in the trial were offered only two 
screens in total and they had not been invited to screening prior to age 70 years. It will be useful to review mortality outcomes after the BreastScreen Australia target age range is extended nationally to 74 years.

Any benefits of screening need to be balanced against potential negative consequences. For example, overdiagnosis needs to be minimised; this study found that higher screening participation by women aged 70-74 years was not associated with a rise in population-level cancer incidence, suggesting that overdiagnosis is minimal in this age group. False positive screening tests (screening mammograms with a positive result, where the screening client is cleared of cancer after further assessment such as further imaging and biopsies) should also be minimised, but they are less likely in this age group than the current target age range: while $10 \%$ of positive screening tests result in a cancer diagnosis for women aged 50 69 years, the figure for women aged $70-79$ years is $18 \%$ [9]). Other potential negative consequences of screening participation include loss of quality of life through earlier cancer diagnosis and treatment, which is increasingly important in developed countries such as Australia where life expectancy is increasing.

A randomised controlled trial of screening in women aged 70 to 74 years would produce the highest quality evidence regarding screening effectiveness. Such a trial could include assessments of cost-effectiveness and quality of life. In the absence of trial data, further observational studies from countries with organised screening programs are needed. In particular, given problems with the recording of nodal status, further analyses of data from programs with more complete information on nodal status would add considerably to the evidence base. 
In conclusion, increased screening participation by women aged 70-74 years is associated with reduced tumour size and possibly reduced nodal involvement at diagnosis without a concomitant increase in cancer incidence. This suggests that extension of age-targetted screening to this age group - as recently done by BreastScreen Australia - is beneficial and can be done without a concern of increased overdiagnosis. 
Acknowledgements: This analysis was originally commissioned by BreastScreen Victoria, with additional funds from National Health and Medical Research Council of Australia (Project Grant 509357). BreastScreen Victoria played no part in the study beyond providing funding. Ethical approval was not required because the study used secondary, population-level aggregate data.

Ethics: This study complies with the National Statement on Ethical Conduct in Human Research (2007) (updated 2009) as per the National Health and Medical Research Council Act 1992.

Conflict of interest: AK and CN initially conducted this analysis through funding provided by BreastScreen Victoria.

Contributors: $\mathrm{CN}$ devised the study and collected the data. $\mathrm{CN}$ and $\mathrm{KM}$ analysed the data and drafted and revised the paper. AK advised on data analysis and revised the paper. 


\section{References}

1. International Agency for Research on Breast Cancer. IARC handbooks of cancer prevention. Vol 7: Breast cancer screening. Lyon: International Agency for Research on Breast Cancer; 2002.

2. Kerlikowske K, Salzmann P, Phillips KA, Cauley JA, Cummings SR. Continuing screening mammography in women aged 70 to 79 years: impact on life expectancy and cost-effectiveness. JAMA: The Journal Of The American Medical Association. 1999;282(22):2156-63.

3. International Cancer Screening Network. Organization of Breast Cancer Screening Programs in 27 ICSN Countries, $2007-2008$. International Cancer Screening Network; 2011; Available from: http://appliedresearch.cancer.gov/icsn/breast/screening.html.

4. Australian Institute of Health and Welfare. Breast cancer in Australia: an overview, 2009 (Cat. no. CAN 46). Canberra: Australian Institute of Health and Welfare, October 2009. Report No.: Cancer series no. 50.

5. Australian Government Department of Health and Ageing. Evaluation of the BreastScreen Australia Program - Evaluation Final Report. Canberra: Australian Government Department of Health and Ageing, 2009.

6. Australian Electoral Commission. Australian Electoral Commission Annual Report 2004-05. Canberra: Australian Electoral Commission, 2005.

7. Australian Institute of Health and Welfare. BreastScreen Australia monitoring report 2005-2006. Cancer series no. 48. Cat. no. CAN 44. Canberra: AIHW, 2009.

8. Hon. Tanya Plibersek, MP. Minister for Health. Australian Government Department of Health and Ageing.Media Release: More women to get free breast cancer screening. Available from:

www.health.gov.au/internet/ministers/publishing.nsf/Content/12142E13A1AE43B0CA257B6A0001FD5F/\$File/TP039.pdf.

9. Department of Health and Ageing \& Australian Institute of Health and Welfare. BreastScreen Australia monitoring report 2004-2005. Canberra: Australian Institute of Health and Welfare (Cancer Series no. 42. Cat no. 37), May 2008.

10. Australian Institute of Health and Welfare \& National Breast Cancer Centre. Breast cancer survival by size and nodal status in Australia. Canberra: Australian Institute of Health and Welfare, 2007.

11. BreastScreen Victoria. BreastScreen Victoria Annual Statistical Report, 2007. Melbourne: BreastScreen Victoria, 2012.

12. Australian Institute of Health and Welfare. BreastScreen Australia monitoring report 2009-10. Cancer series no. 72 . Cat. no. CAN 68. Canberra: AIHW, 2012. 
13. Australian Institute of Health and Welfare. BreastScreen Australia monitoring report 1998-1999 and 1999-2000. Canberra: Australian Institute of Health and Welfare (Cancer Series no. 26. Cat. no. CAN 21), 2003.

14. Australian Institute of Health \& Welfare. BreastScreen Australia monitoring report 2000-2001. Canberra: Australian Institute of Health and Welfare (Cancer Series no. 25. Cat no. CAN 20), 2003.

15. Australian Institute of Health \& Welfare. BreastScreen Australia monitoring report 2001-2002. Canberra: Australian Institute of Health and Welfare (Cancer Series no. 29. Cat no. CAN 24), 2005.

16. Australian Institute of Health \& Welfare. BreastScreen Australia monitoring report 2002-2003. Canberra: Australian Institute of Health and Welfare (Cancer Series no. 32. Cat no. CAN 27), 2006.

17. Department of Health and Ageing \& Australian Institute of Health and Welfare. BreastScreen Australia monitoring report $2003-2004$. Canberra: Australian Institute of Health and Welfare (Cancer series no. 36. Cat. no. CAN 31), 2007.

18. Roder D, Houssami N, Farshid G, Gill G, Luke C, Downey P, et al. Population screening and intensity of screening are associated with reduced breast cancer mortality: evidence of efficacy of mammography screening in Australia. Breast cancer research and treatment. 2008;108(3):409-16.

19. Tabar L, Fagerberg G, Chen HH, Duffy SW, Smart CR, Gad A, et al. Efficacy of breast cancer screening by age. New results from the Swedish Two-County Trial. Cancer. 1995;75(10):2507-17. Epub 1995/05/15. 
Table 1. Observed 2004 screening participation and cancer incidence according to re-invitation policy and State/Territory, for women aged 70-74 years. Screening participation is also shown for women aged 65-69 years, who were consistently re-invited to screening in all jurisdictions during this period.

\begin{tabular}{|c|c|c|c|c|c|c|c|c|c|}
\hline & \multicolumn{5}{|c|}{ Policy in 2004: No re-invitation up to 74 years (re-invitation ceased at 69 years) } & \multicolumn{4}{|c|}{ Policy in 2004: Re-invitation up to 74 years } \\
\hline & $\mathrm{ACT}$ & NSW & SA & WA & Combined & QLD & TAS & VIC & Combined \\
\hline Population, $70-74$ years & 3,793 & 114,224 & 28,464 & 29,191 & 176,696 & 57,448 & 8,674 & 84,161 & 150,283 \\
\hline \multicolumn{10}{|l|}{$\begin{array}{l}\text { Screening participation } \\
(2004 / 2005)\end{array}$} \\
\hline 65-69 years & $0.60(0.59,0.61)$ & $0.54(0.54,0.54)$ & $0.62(0.62,0.63)$ & $0.58(0.58,0.59)$ & $0.56(0.56,0.56)$ & $0.61(0.60,0.61)$ & $0.59(0.58,0.60)$ & $0.58(0.58,0.59)$ & $0.59(0.59,0.60)$ \\
\hline 70-74 years & $0.21(0.20,0.22)$ & $0.25(0.25,0.25)$ & $0.23(0.23,0.24)$ & $0.20(0.19,0.20)$ & $0.24(0.23,0.24)$ & $0.53(0.53,0.54)$ & $0.48(0.47,0.49)$ & $0.51(0.51,0.51)$ & $0.52(0.51,0.52)$ \\
\hline \multicolumn{10}{|l|}{$\begin{array}{l}\text { Cancer incidence per } \\
100,000(95 \% \text { CI })\end{array}$} \\
\hline All cancers & $475(256,693)$ & $325(292,358)$ & & $339(272,406)$ & $332(302,361)$ & $306(261,352)$ & $450(309,590)$ & $291(255,328)$ & $306(278,334)$ \\
\hline Large cancers & & $144(122,166)$ & & $178(130,227)$ & $151(131,171)$ & $97(72,123)$ & $219(121,317)$ & $107(85,129)$ & $110(93,127)$ \\
\hline $\begin{array}{l}\text { Cancers with nodal } \\
\text { involvement }\end{array}$ & & & & $99(63,135)$ & $99(63,135)$ & $70(48,91)$ & $81(21,140)$ & $77(58,96)$ & $75(61,88)$ \\
\hline
\end{tabular}


Table 2. The association between screening participation (change with each absolute $10 \%$ increase in participation) and state-level breast cancer incidence for women aged 70-74 years, for all states/territories and periods with information on tumour size and nodal involvement. ${ }^{\#}$

\begin{tabular}{|l|c|c|}
\cline { 2 - 3 } \multicolumn{1}{c|}{} & \multicolumn{2}{|c|}{$\begin{array}{c}\text { Invasive breast cancer incidence } \\
\text { rate ratio for each absolute } 10 \% \\
\text { increase in participation }\end{array}$} \\
\cline { 2 - 3 } \multicolumn{1}{|c|}{ All invasive breast cancers } & $1.01(0.99$ to 1.03$)$ & 0.222 \\
\hline Large $(>15 \mathrm{~mm})$ breast cancers & $0.92(0.90$ to 0.94$)$ & $<0.001$ \\
\cline { 2 - 3 } & $0.93(0.91 \text { to } 0.96)^{a}$ & $<0.001$ \\
\cline { 2 - 3 } Cancers with nodal involvement & $0.93(0.91 \text { to } 0.95)^{b}$ & $<0.001$ \\
\hline & $0.97(0.95$ to 0.99$)$ & 0.004 \\
\cline { 2 - 3 } & $0.95(0.92 \text { to } 0.97)^{c}$ & $<0.001$ \\
\cline { 2 - 3 } & $1.02(0.98 \text { to } 1.06)^{d}$ & 0.402 \\
\hline
\end{tabular}

\# New South Wales (1997), Queensland (1996-2004), Tasmania (1996-2004), Victoria (1996-2004) and Western Australia (1996-2005).

${ }^{\mathrm{a}-\mathrm{d}}$ Sensitivity analyses:

a. Assuming cancers missing size information are large.

b. Assuming cancers missing size information are small.

c. Assuming cancers missing nodal information have nodal involvement.

d. Assuming cancers missing nodal information have no nodal involvement. 